\title{
A dislodged tooth remained hidden in a Zenker's Diverticulum: A case report
}

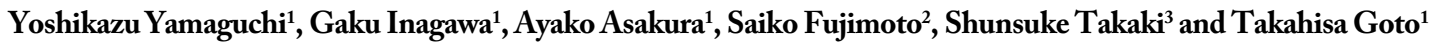 \\ ${ }^{1}$ Department of Anesthesiology and Critical Care, Yokohama City University School of Medicine, Yokohama, Japan \\ ${ }^{2}$ Department of Anesthesiology, Nippon Medical School, Tokyo, Japan \\ ${ }^{3}$ Department of Anesthesiology and Critical Care, Yokohama City University
}

\begin{abstract}
A Zenker's diverticulum is the rarest type of gastrointestinal diverticulum and is located immediately above the upper esophageal sphincter. Here we report the case of a dislodged tooth that entered and remained hidden in a Zenker's diverticulum, and combined use of endoscopy and X-ray fluoroscopy was useful for diagnosis and treatment. To our knowledge, this is the first report of a dislodged tooth located in a Zenker's diverticulum in the perioperative period.
\end{abstract}

\section{Introduction}

Perioperative dental injuries are disturbing events for anesthesiologists, and they occur in $0.02-0.07 \%$ of cases 1 . It is extremely important to locate a dislodged tooth after injury because dental fragments have the potential to perforate the digestive tract. A Zenker's diverticulum (ZD) is the rarest type of gastrointestinal diverticulum and is located immediately above the upper esophageal sphincter. Here we describe the case of a patient with a dislodged tooth that entered and remained hidden in a ZD. The tooth was successfully detected with endoscopy performed under fluoroscopic guidance but not with endoscopy alone. To our knowledge, this is the first report of a dislodged tooth located in a $\mathrm{ZD}$ in the perioperative period.

\section{Case report}

A 73-year-old man was admitted to our hospital with disturbance of consciousness and was diagnosed with acute exacerbation of a chronic subdural hematoma. Surgical evacuation of the hematoma was planned. He had a history of liver cirrhosis, mitral valve regurgitation, hypertension, and prostate carcinoma; however, a ZD was not diagnosed. On physical examination, it was noted that several of his teeth were decaying.

Anesthesia was induced with propofol, fentanyl, and rocuronium. However, tracheal intubation was difficult, and was performed with the intubating laryngeal mask airway. After intubation, careful inspection of the mouth confirmed that no teeth had been damaged. Anesthesia was maintained with sevoflurane, fentany [1], and remifentanil. The surgery was successfully completed, and the trachea was extubated. The anesthesiologist then suctioned the oral cavity; however, one tooth was dislodged during suctioning. Despite several attempts to grasp the tooth with a pair of forceps, the tooth disappeared into the throat. Bronchoscopy revealed that the tooth was neither in the trachea nor in the bronchus. A chest radiograph and computed tomography image showed that the tooth was in the esophagus, at the level of the first rib (Figure 1). We believed that the tooth would naturally move distally and be discharged from the digestive tract. However, a chest

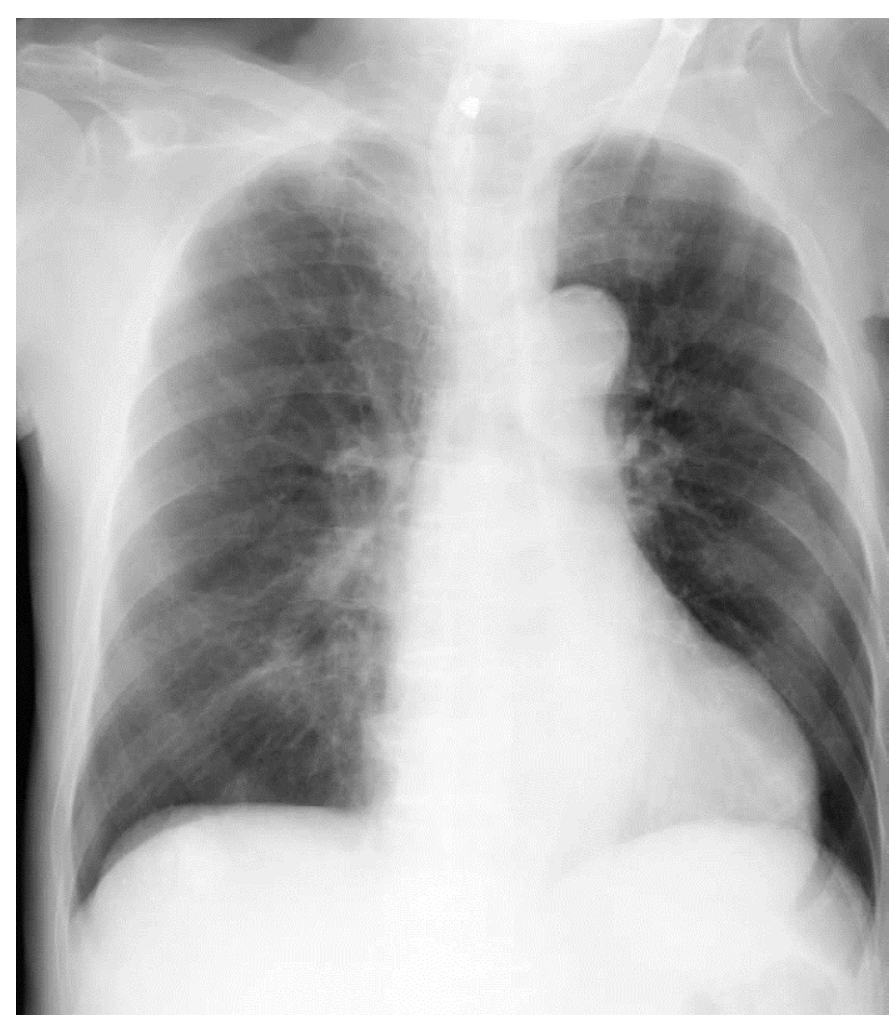

Figure 1. A chest radiograph.

Correspondence to: Yoshikazu Yamaguchi, Department of Anesthesiology and Critical Care Medicine, Yokohama City University School of Medicine, Yokohama, Japan, 3-9 Fukuura, Kanazawa-ku, Yokohama, Kanagawa 236-0004, Japan, Tel: +81-45-787-2918; Fax: +81-45-787-2916; E-mail: yoshikaz@rd6.so-net.ne.jp

Received: May 10, 2015; Accepted: June 10, 2015; Published: June 13, 2015 


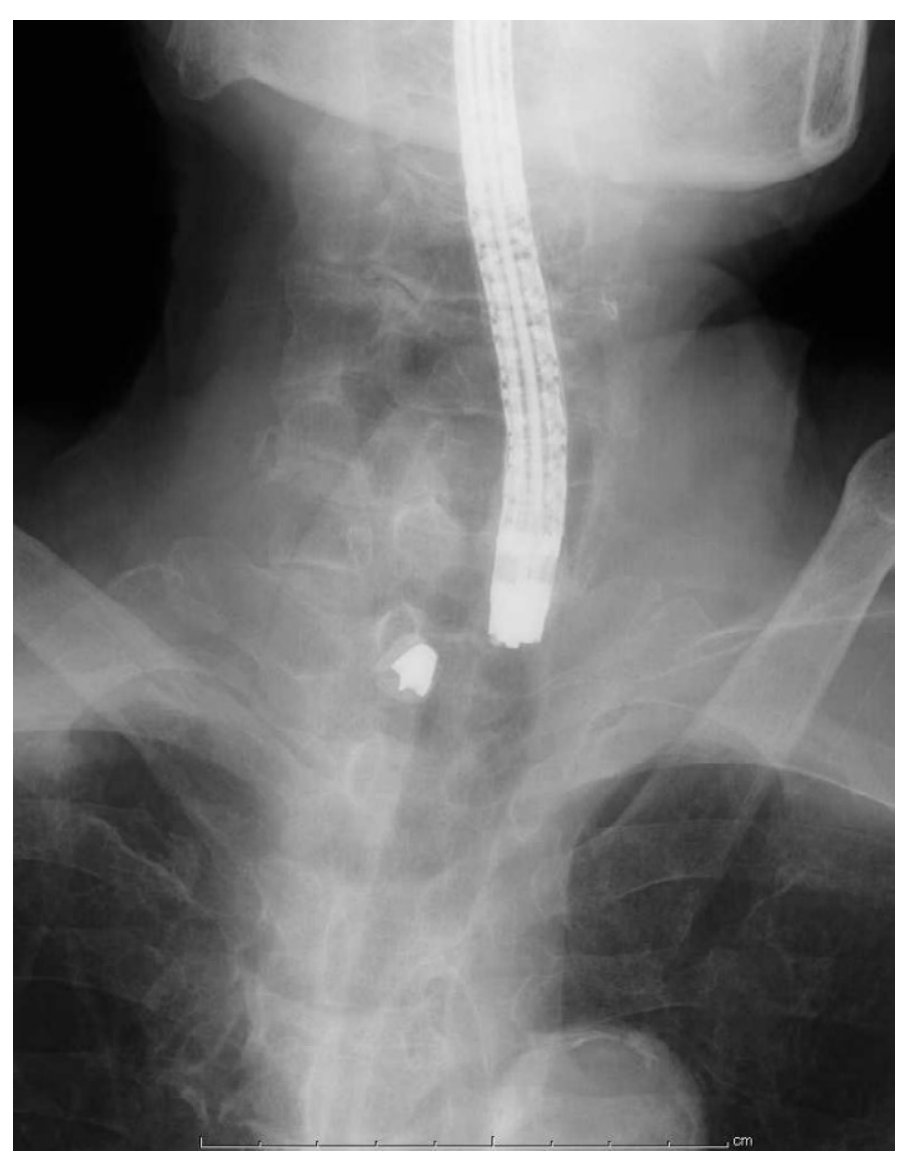

Figure 2. Combined use of endoscopy and X-ray fluoroscopy.

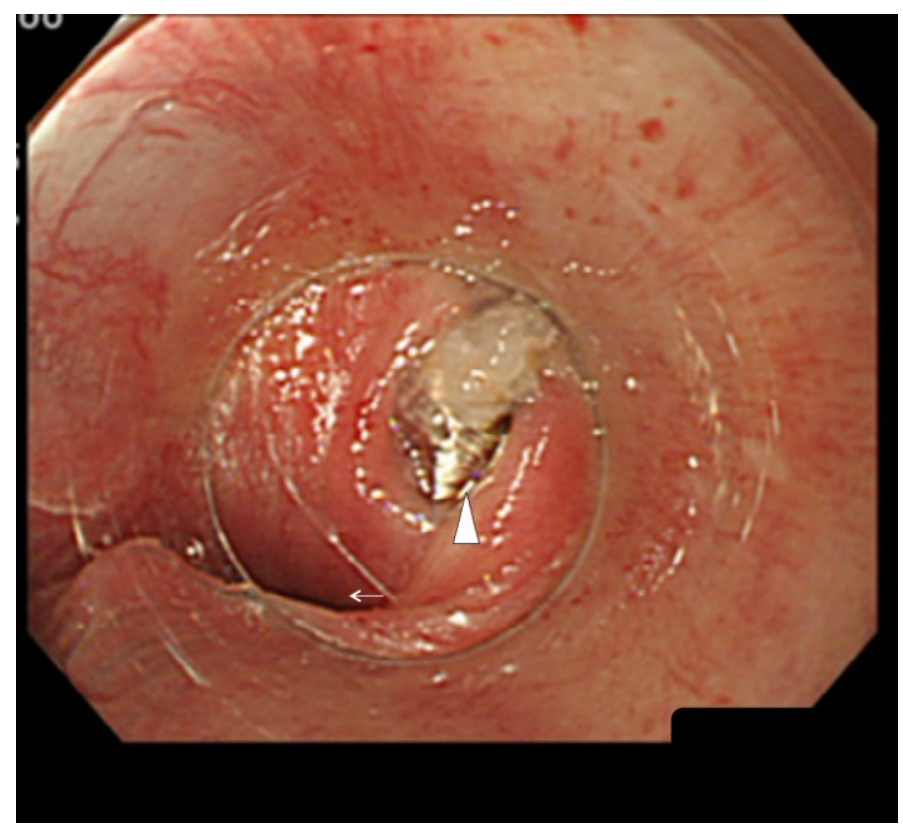

Figure 3. An endoscopic image of the Zenker's diverticulum and the tooth. Arrow head: the Zenker's diverticulum and the tooth. Arrow: the tubular esophagus.

radiograph obtained the next day revealed that the tooth remained at the same location. Endoscopic examination of the esophagus did not detect the tooth at the most likely location indicated by the chest radiograph, and only normal-looking mucosa was observed. Following endoscopic examination, a chest radiograph was obtained after gas insufflation into the esophagus, and no mediastinal gas was noted. Therefore, it was unlikely that the tooth perforated the esophagus and migrated into the mediastinum. Endoscopy was then performed under fluoroscopic guidance to thoroughly examine the esophagus. The tip of the endoscope was positioned as close as possible to the tooth under fluoroscopic guidance, and a transparent plastic tube was used to push the esophageal mucosa at this site. The tooth was then squeezed out from the esophageal wall and was successfully removed. A pouch was identified, and it was diagnosed as a ZD (Figures 2 and 3).

\section{Discussion}

We described the case of a patient with a dislodged tooth that entered and remained hidden in a ZD . A ZD is a posterior diverticulum with a neck proximal to the cricopharyngeal muscle. It is an acquired outpouching of the mucosa through Killian's triangle. A ZD is uncommon, and a community study estimated an annual incidence of 2 per 100,000 people [2]. The majority of patients are old adults (aged $>70$ years) because this condition is asymptomatic in the early stages. Typical symptoms include dysphagia, regurgitation, chronic cough, aspiration, and weight loss [2]. A barium examination is the standard diagnostic procedure.

To our knowledge, this is the first report of a dislodged tooth located in a $\mathrm{ZD}$ in the perioperative period. The frequency of perioperative dental injury is 1 per 2073-2805 anesthesia inductions, and $10.3 \%$ of cases involve a missing tooth or teeth [3]. Our case suggests that, although extremely rare, a missing tooth can remain hidden in a ZD. Because dental fragments have the potential to perforate the digestive tract, every effort should be made to find and, if possible, remove dislodged teeth. The presence of a foreign body other than a tooth in a ZD may be dangerous, and bleeding or ulcers caused by medications retained in a ZD have been reported [4]. As demonstrated in our case, the entry of a ZD can be small and may be overlooked with simple endoscopy. If a tooth or other radiopaque material is present in a $\mathrm{ZD}$, the combined use of endoscopy and X-ray fluoroscopy can detect the foreign object because fluoroscopy permits thorough examination of the esophageal mucosa near the location of a ZD.

If a $\mathrm{ZD}$ is diagnosed preoperatively, the anesthesiologist should be extremely careful to prevent perioperative complications. Aspiration pneumonia and perforation of a $\mathrm{ZD}$ are the major concerns, and these may be life-threatening [5,6]. Anesthetic considerations for a patient with a ZD have been presented in a previous study [7]. First, the anesthesiologist should gather information about the size and position of the pouch opening. Oral premedication should not be administered, because it may be retained in the ZD. Preoperative fasting should be ensured, although fasting does not guarantee an empty ZD. Second, before anesthesia induction, self-induced emptying of the pouch and a head-up tilt of $10-30^{\circ}$ are recommended. Routine cricoid pressure should be avoided, because the cricoid ring is present below the neck of the pouch, and therefore, cricoid pressure may expel the contents of the ZD into the pharynx, which may cause aspiration. Blind insertion of a tracheal tube or nasogastric tube should be avoided as it may cause perforation of the ZD. Therefore, video-assisted laryngoscopy may be useful in patients with a ZD.

Besides a ZD, there are two other types of esophageal diverticula, a traction diverticulum and an epiphrenic diverticulum. A traction diverticulum occurs close to the midpoint of the esophagus. This diverticulum is usually small, and foreign body impaction is rare. To our knowledge, only one case of food impaction in a large traction 
diverticulum has been reported. An epiphrenic diverticulum occurs immediately above the lower esophageal sphincter. This diverticulum is sometimes associated with esophageal achalasia. Unlike in the case of a ZD, these two types of diverticula may benefit from the use of cricoid pressure.

In conclusion, our case demonstrates that it is possible for a dislodged tooth to enter and remain hidden in a ZD.

\section{References}

1. Yasny JS (2009) Perioperative dental considerations for the anesthesiologist. Anesth Analg 108: 1564-1573. [Crossref]

2. Siddiq MA, Sood S, Strachan D (2001) Pharyngeal pouch (Zenker's diverticulum). Postgrad Med J 77: 506-511. [Crossref]
3. Newland MC, Ellis SJ, Peters KR, Simonson JA, Durham TM, et al. (2007) Dental injury associated with anesthesia: a report of 161,687 anesthetics given over 14 years. J Clin Anesth 19: 339-345. [Crossref]

4. Kensing KP, White JG, Korompai F, Dyck WP (1994) Massive bleeding from a Zenker's diverticulum: case report and review of the literature. South Med J 87: 10031004. [Crossref]

5. Aouad MT, Berzina CE, Baraka AS (2000) Aspiration pneumonia after anesthesia in a patient with a Zenker diverticulum. Anesthesiology 92: 1837-1839. [Crossref]

6. Cope R, Spargo P (1990) Anesthesia for Zenker's diverticulum. Anesth Analg 71: 312 [Crossref]

7. Thiagarajah S, Lear E, Keh M (1990) Anesthetic implications of Zenker's diverticulum. Anesth Analg 70: 109-111. [Crossref]

Copyright: $\bigcirc 2015$ Yamaguchi Y. This is an open-access article distributed under the terms of the Creative Commons Attribution License, which permits unrestricted use, distribution, and reproduction in any medium, provided the original author and source are credited. 